\title{
First limits on double beta decays in ${ }^{232} \mathrm{Th}$
}

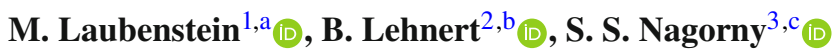 \\ ${ }^{1}$ INFN-Laboratori Nazionali del Gran Sasso, 67100 Assergi, AQ, Italy \\ ${ }^{2}$ Nuclear Science Division, Lawrence Berkeley National Laboratory, Berkeley, CA 94720, USA \\ ${ }^{3}$ Physics Department, Queen's University, Kingston, ON K7L 3N6, Canada
}

Received: 9 April 2020 / Accepted: 23 July 2020 / Published online: 19 August 2020

(C) The Author(s) 2020

\begin{abstract}
As one of the primordial radioactive isotopes, ${ }^{232} \mathrm{Th}$ mainly undergoes $\alpha$-decay with a half-life of $1.402 \cdot 10^{10}$ years. However, it is also one of 35 double beta decay candidates in which the single $\beta$-decay is forbidden or strongly suppressed. $181 \mathrm{mg}$ of thorium contained in a gas mantle were measured in a HPGe well-detector at the Gran Sasso Underground Laboratory with a total exposure of $3.25 \mathrm{~g} \times \mathrm{d}$.

We obtain half-life limits on all double beta decay modes of ${ }^{232} \mathrm{Th}$ to excited states of ${ }^{232} \mathrm{U}$ on the order of $10^{11-15}$ years. For the most likely transition into the $0_{1}^{+}$state we find a lower half-life limit of $6.7 \cdot 10^{14}$ years $(90 \%$ C.I.). These are the first constraints on double beta decay excited state transition in ${ }^{232} \mathrm{Th}$.
\end{abstract}

\section{Introduction}

Double beta decay (DBD) is a second order weak nuclear decay and subject to intense study. While the Standard Model process of two neutrino double beta $(2 \nu \beta \beta)$ decay is experimentally observed in 11 out of 35 possible DBD nuclides $[1,2]$, the lepton number violating process of zero neutrino double beta $(0 \nu \beta \beta)$ decay remains elusive to date. The latter would have profound implications for particle physics and cosmology, implying the Majorana nature of the neutrino and allowing to understand the matter-antimatter asymmetry in the Universe via Leptogenesis [3].

Even though the $2 \nu \beta \beta$ and $0 \nu \beta \beta$ modes require fundamentally different physics, they are connected through the same experimental techniques and share common challenges for nuclear theory. In order to interpret experimentally measured decay rates as a new lepton number violating process, nuclear matrix elements (NME) are required which are notoriously

\footnotetext{
a e-mail: matthias.laubenstein@lngs.infn.it

b e-mail: bjoernlehnert@lbl.gov (corresponding author)

c e-mail: sn65@queensu.ca (corresponding author)
}

difficult to calculate. These calculations can be improved and tested by any additional experimental information of observable $2 v \beta \beta$ decays [4].

The most likely transition for DBD is into the ground state of the daughter nucleus which is typically a $0^{+}-0^{+}$ transition. However, if the Q-value of the isotope is large enough, also transitions into excited state can occur. The measurement of the ground and excited state decay rates in the same nucleus are especially useful for testing nuclear models. Comparing both rates cancels many poorly constraint model parameters and allows for a more direct test of nuclear theory [5].

The end of 20th century and the first quarter of the 21st century could be considered as a "golden age" for direct counting experiments looking for DBD. Many experiments exploiting various detector techniques were proposed and realized within this time period. The highest sensitivities were achieved with the "source $=$ detector" approach, where the isotope of interest is embedded into the material of the detector. In most cases, the experimental signature is the simple sum energy of the two electrons even though some techniques aim at more advanced topology identification [6]. Leading experiments reach half-life limits and sensitivities of over $10^{26}$ years $[7,8]$. However, this way only certain DBD isotopes can be investigated which occur in elements suitable for a working detector technology.

On the other hand, the "source $\neq$ detector" approach, where e.g. a sample containing the isotope of interest is placed on a High Purity Germanium (HPGe) detector, can be applied to searches for DBD in virtually any isotope. The ground state transitions are not accessible with this technique and the experimental signature are the de-excitation $\gamma$-rays from excited state transitions. Consequently, the $2 \nu \beta \beta$ and $0 v \beta \beta$ modes cannot be distinguished since the electrons remain in the external sample ${ }^{1}$. Such experiments have typ-

\footnotetext{
$\overline{1 \text { An exception }}$ are the NEMO and SuperNEMO experiments in which a thin target foil is sandwiched between ionization chambers [9].
} 
ically a smaller detection efficiency, not exceeding a few $\%$, and about two orders of magnitudes lower sensitivity but benefit from very unique experimental signatures of multiple $\gamma$-rays. The best limit with this technique was achieved for the DBD of ${ }^{82} \mathrm{Se}$ to the first excited $0_{1}^{+}$state with $T_{1 / 2}>3 \cdot 10^{22}$ years [10]. Also a combination of these two concepts is used in large scale segmented "source $=$ detector" experiments such as GERDA, CUORE and CUPID0 where the decay occurs in one detector and the $\gamma$-rays are detected in another. These searches have half-life sensitivities of about $10^{23-24}$ years [11-13], but are again limited to certain isotopes within the detection technique.

Measuring samples on a HPGe detector in the "source $\neq$ detector" approach resulted in the first and only $2 v \beta \beta$ decay transitions into excited states in ${ }^{150} \mathrm{Nd}$ and ${ }^{100}$ Mo with measured world average half-lives of $1.33_{-0.26}^{+0.45} \cdot 10^{20}$ years and $5.9_{-0.6}^{+0.8} \cdot 10^{20}$ years, respectively [14].

Lower limits for excited state transitions in other DBD isotopes which were established in Pd isotopes [15], Ce isotopes [16], ${ }^{94} \mathrm{Zr}$ [17], ${ }^{162} \mathrm{Er} \mathrm{[18],}{ }^{168} \mathrm{Yb}$ [19], Sm isotopes $[20],{ }^{74} \mathrm{Se}[21]$, and ${ }^{174} \mathrm{Hf}[22]$ within the last 5 years with half-life values in the range from $10^{17}$ to $10^{20}$ years.

Most of the investigated isotopes are "classical" DBD emitters, where a nucleus $A(Z, N)$ cannot undergo single beta-decay to $A(Z \pm 1, N \pm 1)$ because it is energetically forbidden or heavily suppressed by an unfavorable isospin configuration. However, some of these classical DBD emitters can decay via other modes. Recent measurements with a platinum sample demonstrated a search for DBD in the unstable ${ }^{190} \mathrm{Pt}$ [23], which has a more favorable decay through conventional $\alpha$-decay with significantly shorter half-life. Systems with $\beta \beta$-processes in unstable nuclides were discussed in [24], where lower limits on DBD of primordial ${ }^{235} U,{ }^{238} U$, ${ }^{232} \mathrm{Th}$ nuclides and their daughters were established. The authors have been utilizing long term low-background measurements with $\mathrm{CaWO}_{4},{ }^{116} \mathrm{CdWO}_{4}$ and $\mathrm{Gd}_{2} \mathrm{SiO}_{5}$ scintillating crystals for these analyses. The isotopes of interest were determined as internal contamination of these scintillating crystals. Despite a very low concentration of the isotopes of interest, half-life limits in the range $10^{11-12}$ years were set for the first time for the $0 \nu \beta \beta$ and $2 \nu \beta \beta$ decay modes to the ground state.

In this work we investigate DBD of ${ }^{232} \mathrm{Th}$ with the "source $\neq$ detector" approach using HPGe $\gamma$-spectroscopy. Thorium is a mono-isotopic element and thus, the isotopic abundance of ${ }^{232} \mathrm{Th}$ is $100 \%$ in natural thorium. The single $\beta$-decay of ${ }^{232} \mathrm{Th}$ to ${ }^{232} \mathrm{~Pa}$ is energetically forbidden but the $\alpha$-decay to ${ }^{228} \mathrm{Ra}$ is possible with $1.402 \cdot 10^{10}$ years half-life. DBD of ${ }^{232} \mathrm{Th}$ is possible into the ground state as well as into a variety of excited states of ${ }^{232} \mathrm{U}$. The possible decay modes are illustrated in Fig. 1. The most likely excited state transition is the $0_{1}^{+}$state at $691.4 \mathrm{keV}$. To our knowledge there were no previous attempts to search for DBD excited state transitions in ${ }^{232} \mathrm{Th}$.

There are some theoretical half-live predictions for ${ }^{232} \mathrm{Th}$ of which we list those appearing in the literature after the year 2000 . The $2 \nu \beta \beta$ ground state transition is predicted with halflives of 5.3 $\cdot 10^{22}$ years [26], $4.2 \cdot 10^{21}$ years [25] and 1.9 . $10^{22}$ years [4]. The $0 \nu \beta \beta$ ground state transition, assuming a $1 \mathrm{eV}$ Majorana neutrino mass, is predicted at $5.4 \cdot 10^{24}$ years [26] and $4.4 \cdot 10^{23}$ years [4]. For excited state transitions there exist predictions for the $2_{1}^{+} 47.6 \mathrm{keV}$ state at $1.4 \cdot 10^{30}$ years $(2 \nu \beta \beta)[27]$ and for the $0_{1}^{+} 691.4 \mathrm{keV}$ state $6.1 \cdot 10^{30}$ years $(2 v \beta \beta)$ and $1.7 \cdot 10^{28}$ years $\left(0 v \beta \beta, m_{\beta \beta}=1 \mathrm{eV}\right)[4]$.

\section{Sample and experimental setup}

The measurement of a gas mantle sample containing 0.1811(5) g thorium was carried out in the STELLA (SubTerranean Low Level Assay) facility in the underground laboratories of LNGS (Laboratori Nazionali del Gran Sasso) of INFN in Assergi, Italy, which provided an average shielding of $\approx 3600$ meter of water equivalent . Details can be found in $[29,30]$. The sample was placed in a $1 \mathrm{ml}$ plastic vial with a cone-shaped bottom, then vacuum sealed in two plastic bags and placed into the well of an ultra low-background high purity germanium (HPGe) well-type detector. The HPGe detector, named GeDSG, has a $35.2 \%$ efficiency relative to a $3 \times 3$ in $\mathrm{NaI}(\mathrm{Tl})$ crystal scintillator and a thin $0.75 \mathrm{~mm}$ aluminum window [29]. The detector is surrounded by a composite shield starting on the outside with $10 \mathrm{~cm}$ low activity lead $\left(<100 \mathrm{~Bq} / \mathrm{kg}\right.$ of $\left.{ }^{210} \mathrm{~Pb}\right)$, followed by another $5 \mathrm{~cm}$ of even lower activity lead $\left(<6 \mathrm{~Bq} / \mathrm{kg}\right.$ of $\left.{ }^{210} \mathrm{~Pb}\right)$ and then $5 \mathrm{~cm}$ of oxygen-free high conductivity (OFHC) copper, exposed only for a very short time to cosmic rays above ground. Finally, the shield and detector are enclosed in an air tight housing kept at slight overpressure and continuously flushed with boil-off from liquid nitrogen to prevent and remove radon gas from the setup. An illustration of the setup is shown in Fig. 2.

The energy spectrum of the thorium sample was accumulated over $378.1 \mathrm{~h}$, and is presented in Fig. 3. The trigger rate of the detector was $800 \mathrm{~Hz}$ and thus, higher than for typical low-background measurements. The dead-time due to random pile-up in the $50 \mu$ s trigger window is about $5 \%$. It is accounted for in the analysis through the livetime of the dataset. Small non-Gaussian tails can be observed for the peaks in the spectrum. They affect the event counting by less than $2 \%$ and the determination of the mean peak energy by less than $0.2 \mathrm{keV}$. Since these effects are negligible and accounted for in the systematics of the fit, we proceed using a simple Gaussian peak shape for the analysis. We use prominent $\gamma$-lines in the ${ }^{232} \mathrm{Th}$ spectrum for in-situ calibration of energy and resolution in order to avoid small changes due to the high rate compared to standard calibrations with ${ }^{241} \mathrm{Am}$, 
Fig. 1 Decay scheme of all possible ${ }^{232}$ Th double beta decay transitions. The $0^{+}$ transitions are highlighted. Data taken from [28]

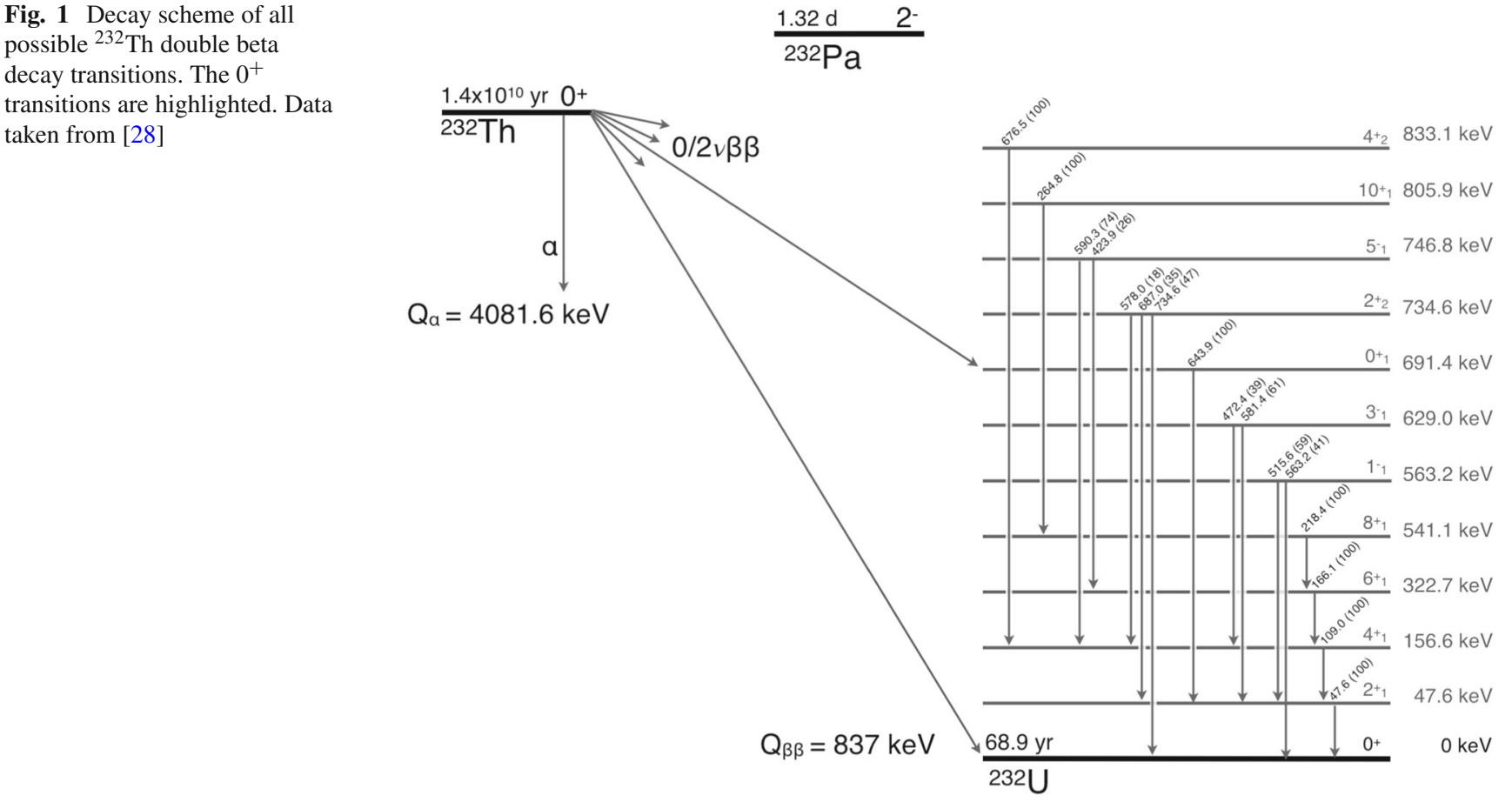

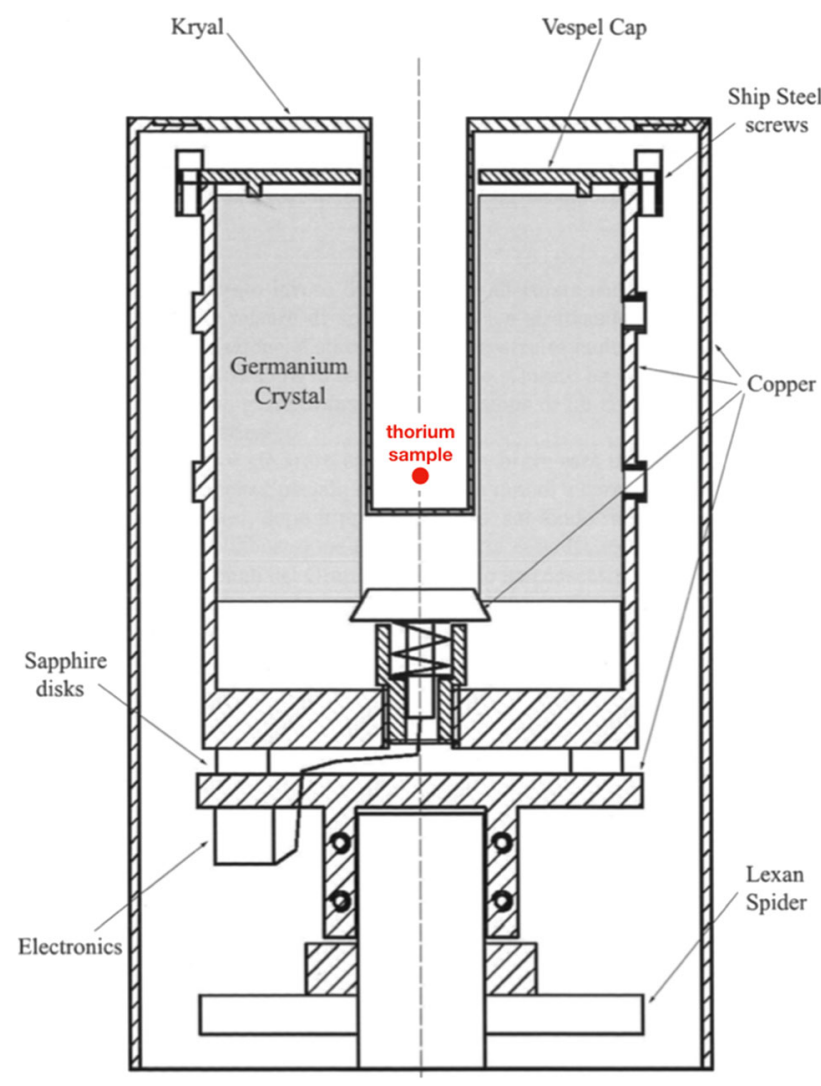

Fig. 2 Setup and sample configuration of the measurement. Figure adopted from [29]
${ }^{133} \mathrm{Ba}$ and ${ }^{60} \mathrm{Co}$ sources. The energy resolution function is $\sigma_{E}=1.40+9.89 \cdot 10^{-5} E+1.82 \cdot 10^{-8} E^{2}$ and shows no significant difference for the thorium dataset compared to standard calibrations. Small difference in resolution are observed at lower energies which are taken into account in the in-situ calibration. The efficiencies for the full-energy absorption peaks used for the quantitative analysis were obtained by Monte-Carlo simulation with the MaGe code based on the GEANT4 software package [31,32].

\section{Analysis}

The analysis is based on peak searches for de-excitation $\gamma$ rays from the various excited state decay modes. The full spectrum is shown in Fig. 3 (left). The high trigger rate of the detector results in $10^{4-6}$ counts per $\mathrm{keV}$ which requires the search for rare events on top of a large background. The background expectation in such searches is typically taken from a background model built by Monte-Carlo simulations or empirically by assuming a linear behavior around the peak. However, given the large number of events per bin, the background expectation requires per cent or even per mille precision which is not realistically achievable. Figure 3 (right) shows a zoom into the spectrum around the $643.5 \mathrm{keV} \gamma$-line of the $0_{1}^{+}$transition clearly indicating that a linear behavior cannot be assumed with the required precision. Thus, we obtain count limits of the signal peaks by excluding Gaussian peak shapes on top of the observed number of events without assuming an a-priori background. This method does 

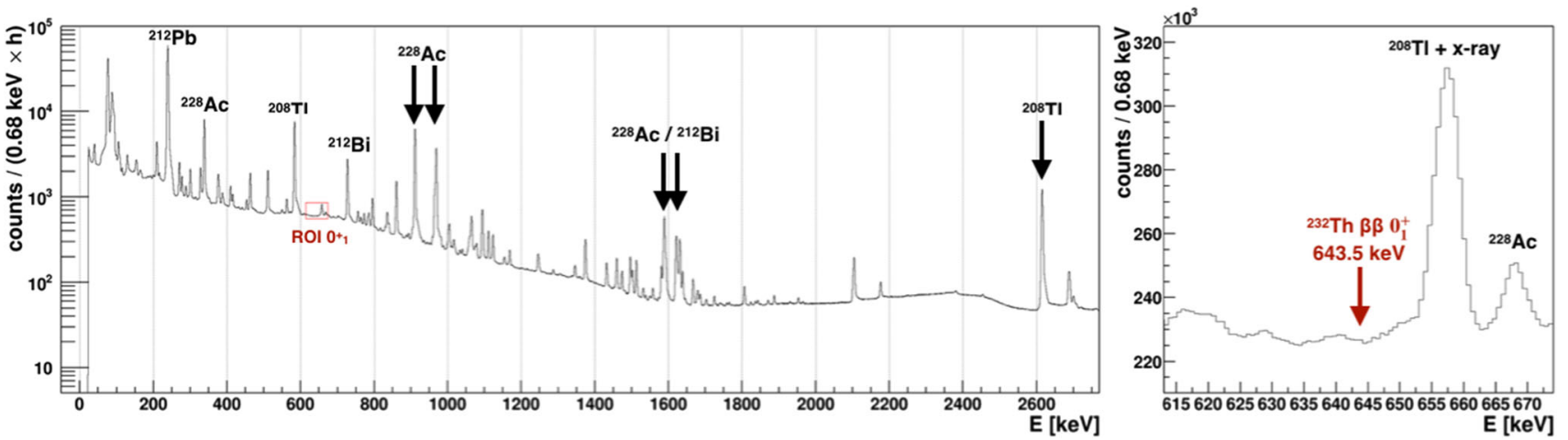

Fig. 3 Measured HPGe spectrum of the $0.181 \mathrm{~g}$ thorium sample obtained in 16.6 days (15.8 days livetime). Left: full spectrum with prominent $\gamma$-lines highlighted. Right: zoom in to the region of interest for the $643.5 \mathrm{keV} \gamma$-line of ${ }^{232} \mathrm{Th}$ double beta decay transition to the $0_{1}^{+}$ state. Significant spectral substructures are clearly visible in-between the main peaks due to the large number of recorded events not allow to discover a signal since all observed events are interpreted as background.

The peak fits are performed in a Bayesian regime for all possible double beta decay modes of ${ }^{232} \mathrm{Th}$ individually. Here, the analysis is described for the $0_{1}^{+}$decay mode as example. The likelihood $\mathcal{L}$ is defined as the product of the Poisson probabilities over each bin $i$ for observing $n_{i}$ events while expecting $\lambda_{i}$, in which $\lambda_{i}$ is the sum of the signal $S_{i}$ and background $B_{i}$ expectation:

$$
\mathcal{L}(\mathbf{p} \mid \mathbf{n})=\prod_{i} \frac{\lambda_{i}(\mathbf{p})^{n_{i}}}{n_{i} !} e^{-\lambda_{i}(\mathbf{p})}, \lambda_{i}(\mathbf{p})=S_{i}+B_{i}
$$

Here $\mathbf{n}$ denotes the data and $\mathbf{p}$ the set of floating parameters.

$S_{i}$ is taken as the integral of the Gaussian peak shape in this bin given the total signal peak counts $s$

$S_{i}=\int_{\Delta E_{i}} \frac{s}{\sqrt{2 \pi} \sigma_{E}} \cdot \exp \left(-\frac{\left(E-E_{0}\right)^{2}}{2 \sigma_{E}^{2}}\right) d E$

where $\Delta E_{i}$ is the bin width, $\sigma_{E}$ the energy resolution and $E_{0}$ the $\gamma$-line energy as the mean of the Gaussian.

$B_{i}$, the background expectation, is implemented as a free parameter for each bin with a Gaussian prior with mean $n_{i}$ and width $\sqrt{n_{i}}$

$B_{i}=n_{i} \cdot \frac{1}{\sqrt{2 \pi n_{i}}} \cdot \exp \left(-\frac{\left(b_{i}-n_{i}\right)^{2}}{2 n_{i}}\right)$.

This method adds an additional fit parameter $b_{i}$ for each bin but correctly distinguishes between the background expectation in the fit $B_{i}$ and the observed numbers of events $n_{i}$ on which the expectation is based. The best fit for $b_{i}$ will be identical to $n_{i}$, but the additional degrees of freedom widen the posterior distribution and result in half-life limits which are about $30 \%$ more conservative compared to simply fixing $B_{i} \equiv n_{i}$ in the analysis.
The signal counts are connected with the half-life $T_{1 / 2}$ of the decay mode as

$s=\ln 2 \cdot \frac{1}{T_{1 / 2}} \cdot \epsilon \cdot N_{A} \cdot T \cdot m \cdot f \cdot \frac{1}{M}$,

where $\epsilon$ is the full energy peak detection efficiency, $N_{A}$ is the Avogadro constant, $T$ is the live-time ( $15.75 \mathrm{~d}$ ), $m$ is the mass of the sample $(0.181 \mathrm{~g})$, and $f$ is the isotopic fraction of ${ }^{232} \mathrm{Th}(100 \%)$ and $M$ its molar mass (232).

Each free parameter in the fit has a prior associated. The prior for the inverse half-life $\left(T_{1 / 2}\right)^{-1}$ is flat. Priors for energy resolution, peak position and detection efficiencies are Gaussian, centred around the mean values of these parameters. The width of these Gaussians are the uncertainty of the parameter values. This naturally includes the systematic uncertainty into the fit result.

The uncertainty of the peak positions are set to $0.1 \mathrm{keV}$. The energy scale and resolution are obtained with the ${ }^{232} \mathrm{Th}$ decay chain $\gamma$-lines in the spectrum. A resolution of $\sigma=$ $1.48 \mathrm{keV}$ was determined at $643.5 \mathrm{keV}$ with an estimated uncertainty of $10 \%$ which also accounts for slightly nonGaussian peak shapes due to pile-up from the high rate operation. The full energy peak detection efficiencies are determined with Geant4 Monte-Carlo simulations and are 14.9\% at $643.5 \mathrm{keV}$ with an assumed uncertainty of $10 \%$. Systematic uncertainties on the measured sample mass and the isotopic fraction in the sample are negligible with respect to the uncertainty of the detection efficiency.

The posterior probability distribution is calculated from the likelihood and prior probabilities with the Bayesian Analysis Toolkit (BAT) [33] and marginalized for $\left(T_{1 / 2}\right)^{-1}$. The best fit is always zero signal counts in this method since all observed events are consistent with the background by design. The $90 \%$ quantile of the marginalized posterior distribution of $\left(T_{1 / 2}\right)^{-1}$ is used to set the $90 \%$ credibility limits including systematic uncertainties. For the $0_{1}^{+}$transition, 


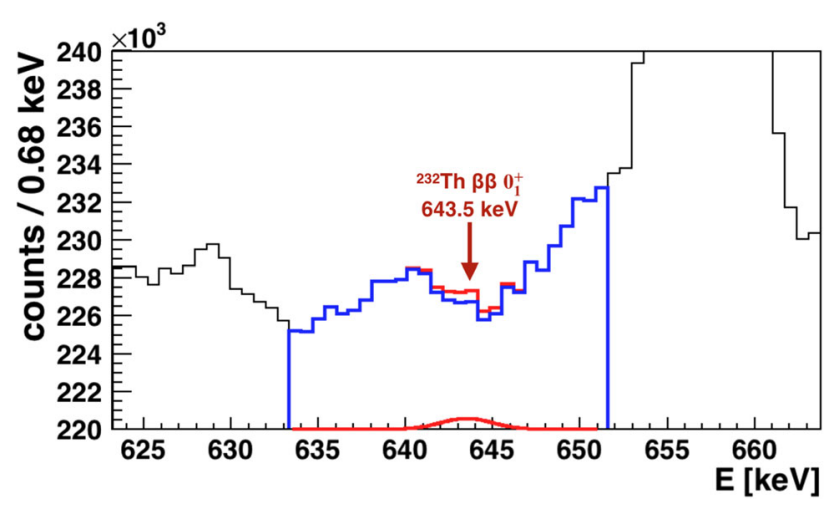

Fig. 4 Region of interest and fit for the $0_{1}^{+}$transition. The data is shown in black. The mean background expectation in each bin identical to the data by construction is shown in blue. The signal peak excluded by $90 \%$ probability is shown in red on of the data as well as independently at the bottom

3145 counts are excluded in the $643.5 \mathrm{keV}$ peak on top of a background of $3.5 \cdot 10^{5} \mathrm{cts} / \mathrm{keV}$. The lower half-life limit is

$T_{1 / 2}>6.7 \times 10^{14}$ years $(90 \% \mathrm{CI})$.

The fit is shown in Fig. 4 illustrating the fit function in red with the signal peak set to the strength excluded with $90 \%$ credibility. The fit function for the best fit, i.e. without signal strength and background equivalent to the observed number of events, is shown in blue.

The other decay modes are treated similarly and results are shown in Table 1. In case multiple $\gamma$-lines are considered, a combined fit is performed by extending the likelihood in Eq. 1 over multiple regions of interest with common $\left(T_{1 / 2}\right)^{-1}$ parameter. The $47.6 \mathrm{keV} \gamma$-line is part of all decay modes but due to its low energy it has only a small detection efficiency and higher background level in the setup. It is only considered for the first excited state $\left(2_{1}^{+}\right)$where it is the only $\gamma$-line and where the obtained half-life limit is about 3 orders of magnitude smaller than for the other modes. The $578.0 \mathrm{keV}$ $\gamma$-line of the $5_{1}^{-}$state is omitted due to its low branching ratio. The complete list of considered $\gamma$-lines for each decay mode is listed in the last column of Table 1 .

\section{Conclusions and outlook}

We performed a first search for double beta decays of ${ }^{232} \mathrm{Th}$ into all possible excited states of ${ }^{232} \mathrm{U}$ using a thorium containing gas mantle sample and a HPGe well detector. The established limits are valid for both the $2 \nu \beta \beta$ and $0 \nu \beta \beta$ modes. The large intrinsic background, dominated by thorium daughter nuclides in the sample, did not allow to model the background prediction with sufficient precision. Thus the analysis was performed without background model and limits on ${ }^{232} \mathrm{Th}$ DBDs were set under the assumption that all observed events are background, i.e. a discovery with this method is not possible.

Future improvements of this measurement should aim at reducing the intrinsic thorium daughter background in the search. This background builds up over time and reaches equilibrium after a few half-lives of ${ }^{228} \mathrm{Ra}\left(\mathrm{T}_{1 / 2}=5.7\right.$ years). Thus the mass and the age of the sample must be well chosen based on the acceptable count rate for the used detector setup. Starting with a chemically pure thorium sample e.g. by anion-exchange resin purification, would allow to limit the total daughter nuclide activity to less than $10 \%$ of the initial ${ }^{232} \mathrm{Th}$ activity within the first 180 days. This is a factor of 100 less intrinsic background compared to the current sample in equilibrium. Instead of reducing the background, this optimization could be used to increase the ${ }^{232} \mathrm{Th}$ sample mass which would allow to improve the half-live sensitivity

Table 1 Lower half-life limits on ${ }^{232}$ Th double beta decay modes set in this work. The last column shows the $\gamma$-lines used in the combine fit along with their detection efficiency in the decay mode.

\begin{tabular}{lll}
\hline $\begin{array}{l}\text { Decay level } \\
{[\mathrm{keV}]\left(J^{\pi}\right)}\end{array}$ & $\begin{array}{l}\mathrm{T}_{1 / 2}(90 \% \mathrm{CI}) \\
{[\text { years] }}\end{array}$ & $\begin{array}{l}\gamma \text {-lines energies (efficiency) } \\
{[\mathrm{keV}]([\%])}\end{array}$ \\
\hline $47.6\left(2_{1}^{+}\right)$ & $>4.8 \cdot 10^{11}$ & $47.6(0.021)$ \\
$156.6\left(4_{1}^{+}\right)$ & $>9.1 \cdot 10^{13}$ & $109.0(4.4)$ \\
$322.7\left(6_{1}^{+}\right)$ & $>3.2 \cdot 10^{14}$ & $109.0(3.1), 166.1(12.6)$ \\
$541.1\left(8_{1}^{+}\right)$ & $>1.8 \cdot 10^{14}$ & $109.0(1.8), 166.1(7.1), 218.4(15.5)$ \\
$563.2\left(1_{1}^{+}\right)$ & $>4.4 \cdot 10^{14}$ & $515.6(10.9), 563.2(7.0)$ \\
$629.0\left(3_{1}^{+}\right)$ & $>3.6 \cdot 10^{14}$ & $109.0(0.8), 472.4(7.5), 581.4(9.8)$ \\
$691.4\left(0_{1}^{+}\right)$ & $>6.7 \cdot 10^{14}$ & $643.5(14.9)$ \\
$734.6\left(2_{2}^{+}\right)$ & $>3.8 \cdot 10^{14}$ & $687.0(5.2), 734.6(6.3)$ \\
$746.8\left(5_{1}^{-}\right)$ & $>1.7 \cdot 10^{14}$ & $109.0(1.9), 166.0(1.5), 424.3(4.0), 590.4(12.2)$ \\
$805.9\left(10_{1}^{+}\right)$ & $>4.1 \cdot 10^{14}$ & $109.0(0.96), 166.0(3.5), 218.4(7.7), 264.8(10.0)$ \\
$833.1\left(4_{2}^{+}\right)$ & $>5.9 \cdot 10^{14}$ & $109.0(2.2), 676.5(13.9)$
\end{tabular}


directly by two-orders of magnitude. Longer measurement campaigns could be achieved by intermittent re-purification of the sample. For a 1 year measurement with an optimized sample, we expect a half-life sensitivity of $3 \cdot 10^{17}$ years for the $0_{1}^{+}$transition, which is competitive to other exotic isotopes.

Faster detectors, such as thorium loaded liquid scintillators in "source = detector" configuration, are less subject to pile-up effects and would allow to accommodate even more ${ }^{232}$ Th nuclei. Even so intermittent re-purification is more difficult in scintillators compared to inert samples, this approach would allow to improve the sensitivity further and additionally enable to investigate the $0 / 2 \nu \beta \beta$ ground state transitions.

Acknowledgements We would like to thank Dr. Fedor Šimkovic for interesting and useful discussion about theoretical half-life estimates.

Data Availability Statement This manuscript has no associated data or the data will not be deposited. [Authors' comment: Data are available from the corresponding authors upon reasonable request.]

Open Access This article is licensed under a Creative Commons Attribution 4.0 International License, which permits use, sharing, adaptation, distribution and reproduction in any medium or format, as long as you give appropriate credit to the original author(s) and the source, provide a link to the Creative Commons licence, and indicate if changes were made. The images or other third party material in this article are included in the article's Creative Commons licence, unless indicated otherwise in a credit line to the material. If material is not included in the article's Creative Commons licence and your intended use is not permitted by statutory regulation or exceeds the permitted use, you will need to obtain permission directly from the copyright holder. To view a copy of this licence, visit http://creativecomm ons.org/licenses/by/4.0/.

Funded by SCOAP ${ }^{3}$.

\section{References}

1. V.I. Tretyak, Y.G. Zdesenko, Atom. Data Nucl. Data Tabl. 80, 83$116(2002)$

2. N. Ackerman et al., EXO-200 Collaboration. Phys. Rev. Let. 107, $212501(2011)$
3. M. Fukugita, T. Yanagida, Phys. Lett. B 174, 45 (1986)

4. J. Barea, J. Kotila, F. Iachello, Phys. Rev. C 91, 034304 (2015)

5. B. Lehnert, EPJ Web Conf. 93, 01025 (2015)

6. F. Monrabal et al., J. Inst. 13, P12010 (2018)

7. A. Gando et al., (KamLAND-Zen Collaboration) Phys. Rev. Lett. 117, 082503 (2016)

8. M. Agostini et al., (GERDA Collaboration) Science 365, 1445 (2019)

9. R. Arnold et al., SuperNEMO Collaboration. Eur. Phys. J. C 70(4), 927 (2010)

10. J.W. Beeman et al., Eur. Phys. J. C 75, 591 (2015)

11. M. Agostini et al., J. Phys. G 42(11), 1-18 (2015)

12. C. Alduino et al., CUORE Collaboration. Eur. Phys. J. C 79, 795 (2019)

13. O. Azzolini et al., Eur. Phys. J. C 78, 888 (2018)

14. A.S. Barabash, Phys. Rev. C 81, 035501 (2010)

15. B. Lehnert et al., J. Phys. G 43, 115201 (2016)

16. P. Belli et al., Eur. Phys. J. A 53, 172 (2017)

17. N. Dokania, D. Degering, B. Lehnert, V. Nanal, K. Zuber, J. Phys. G 45, 075104 (2018)

18. P. Belli et al., J. Phys. G 45, 095101 (2018)

19. P. Belli et al., Nucl. Phys. A 990, 64 (2019)

20. P. Belli et al., Eur. Phys. J. A 55, 201 (2019)

21. A.S. Barabash et al., Nucl. Phys. A 996, 121697 (2020)

22. F.A. Danevich et al., Nucl. Phys. A 996, 121703 (2020)

23. P. Belli et al., Eur. Phys. J. A 47, 91 (2011)

24. V.I. Tretyak, F.A. Danevich, S.S. Nagorny, YuG Zdesenko, Eur. Phys. Lett. 69(1), 41-47 (2005)

25. A.A. Raduta et al., Phys. Rev. C 69, 064331 (2004)

26. J.G. Hirsch et al., Phys. Lett. B 534, 57 (2002)

27. C.M. Raduta, A.A. Raduta, Phys. Rev. C 76, 044306 (2007)

28. E. Browne, Nucl. Data Sheets 107, 2579 (2006)

29. M. Laubenstein, Int. J. Mod. Phys. A 32(30), 174,3002 (2017)

30. C. Arpesella, Appl. Rad. Isot. 47, 991-996 (1996)

31. M. Boswell et al., IEEE-NS 58(3), 1212 (2011)

32. S. Agostinelli et al., Nucl. Instrum. Meth. A 506, 250-303 (2003)

33. A. Caldwell, D. Kollár, K. Kröninger, Comp. Phys. Commun. 180, 2197 (2009) 